\title{
Surfactant addition to alkali solutions for increased carbon dioxide absorption rates
}

\author{
Brett P. Spigarelli \\ Michigan Technological University, Houghton, MI \\ bpspigar@mtu.edu
}

\begin{abstract}
To meet the growing need for $\mathrm{CO}_{2}$ capture and storage technology, Michigan Technological University is studying CO capture using alkali-based solvents. The objective of the present study was to find a way to increase the absorption rate of $\mathrm{CO}_{2}$ into an alkali solution without reducing the absorption capacity of the solution. One approach to increasing absorption rate is to use a surfactant to chemically alter the gas bubble size. The surfactant chosen for the study was a neutral-charge polypropylene glycol methyl ether (PPGME) with a molecular weight of 200. Experiments were conducted to study the absorption rate of $\mathrm{CO}_{2}$ at varying surfactant concentrations. Results showed that as the concentration of $P P G M E$ increased in solution, the absorption rate also increased. The $\mathrm{CO}_{2}$ absorption rate increased from $3.45 \times 10^{-3}$ $\mathrm{mol} / \mathrm{min} \mathrm{CO}_{2}$ at $\mathrm{Og} / \mathrm{L}$ PPGME to $3.92 \times 10^{-3} \mathrm{~mol} / \mathrm{min} \mathrm{CO}_{2}$ at $0.48 \mathrm{~g} / \mathrm{L} \mathrm{PPGME}$. This amounted to a $14 \%$ increase in the $\mathrm{CO}_{2}$ absorption rate with no decrease in the absorption capacity of the solution.
\end{abstract}

Key words: Carbon dioxide, Capture, Absorption, Surfactant

\section{Introduction}

Chemical absorption using an amine solvent is currently the most mature carbon capture and storage technology available. Amines are advantageous in that they have a fast $\mathrm{CO}_{2}$ absorption rate, which leads to a high $\mathrm{CO}_{2}$ removal efficiency. However, amine absorption has some considerable drawbacks, such as high energy requirements, high equipment corrosion rates, amine degradation in the presence of oxygen, high waste generation and a large increase in plant footprint if new equipment is constructed (Herzog and Golomb, 2004; D'Alessandro et al., 2010). Due to these drawbacks, Michigan Technological University (MTU) is studying $\mathrm{CO}_{2}$ absorption using alkali-based solvents, which address the above disadvantages of amine solvents.

However, the $\mathrm{CO}_{2}$ absorption rate in alkali solvents (e.g., a potassium carbonate solution) is perceived to be slow in comparison to amine solvents. It is believed that rate-increasing additives will need to be identified for $\mathrm{CO}_{2}$ capture with alkali solvents to be feasible. The focus of this study is to identify a surfactant that can increase the $\mathrm{CO}_{2}$ absorption rate into an alkali solution without adversely impacting the absorption capacity of the solution. For the present study, $\mathrm{CO}_{2}$ was bubbled through an alkali solution with varying concentrations of a polypropylene glycol methyl ether (PPGME). By knowing the inlet and outlet volumetric flowrates of $\mathrm{CO}_{2}$, the moles of $\mathrm{CO}_{2}$ absorbed was calculated as the difference between moles of $\mathrm{CO}_{2}$ in the exhaust stream and the moles of $\mathrm{CO}_{2}$ in the feed stream. The PPGME surfactant was chosen because it reduces the gas bubble size distribution and average bubble diameter. This would thereby increase the gas-liquid interfacial area, in theory increasing the $\mathrm{CO}_{2}$ absorption rate. Thus, objectives of the study include the following: 1) examine the effect of PPGME on the $\mathrm{CO}_{2}$ absorption rate and 2) examine the effect of PPGME on the $\mathrm{CO}_{2}$ absorption capacity of the solution.

\section{Results and discussion}

Experiments were conducted to study the absorption rate of $\mathrm{CO}_{2}$ in the alkali solvent at varying surfactant concentrations of $0,0.12,0.24,0.36$ and $0.48 \mathrm{~g} / \mathrm{L}$. Results show that the addition of
PPGME to the alkali solution increased the absorption rate of $\mathrm{CO}_{2}$. Initially, at $0.12 \mathrm{~g} / \mathrm{LPPGME}$, a significant increase in absorption rate was seen. However, above $0.12 \mathrm{~g} / \mathrm{L}$ PPGME, diminishing returns were seen with respect to increasing the absorption rate. This shows that a substantial increase in absorption rate can be achieved at a relatively low surfactant concentration. Results also show a negligible decrease in the $\mathrm{CO}_{2}$ absorption capacity of each solution. The absorption capacity of each solution can be seen in Fig. 1 as the last data point on each curve.

\section{References}

D'Alessandro, D.M., Smit, B., and Long, J.R., 2010, "Carbon dioxide capture: prospects for new materials," Angewandte Chemie, International Edition, Vol. 49, pp. 6058-6082.

Herzog, H., and Golomb, D., 2004, "Carbon capture and storage from fossil fuel use," Encyclopedia of Energy, Vol. 1, pp. 1-11.

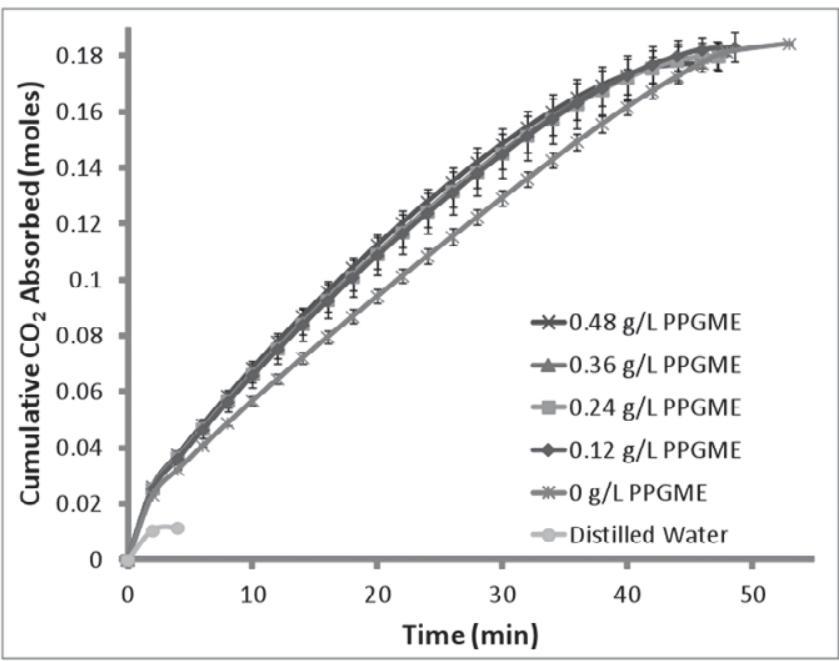

Figure $1-\mathrm{CO}_{2}$ absorption in a $2 \%(\mathrm{w} / \mathrm{w})$ alkali solution with varying concentrations of PPGME in solution. As the concentration of PPGME increased, the absorption rate of $\mathrm{CO}_{2}$ also increased, and the $\mathrm{CO}_{2}$ absorption capacity of the solution remained constant. Trials were repeated in triplicate. The error bars represent one standard deviation from the average. 